\title{
Reliable Data Collection in EWSN based Wireless Sensor Network
}

\author{
Pradeep K Deshmukh, \\ Ph.D \\ Professor \\ Rajashri Shahu College of \\ Engg. Pune
}

\author{
Suruchi S Nannaware \\ Student \\ Rajashri Shahu College of \\ Engg. Pune
}

\author{
Rajashri Deshmukh \\ Asso. professor \\ Rajashri Shahu College of \\ Engg. Pune
}

\begin{abstract}
The recent development in wireless technology, has led to reduce the problems in the day to day life. Similarly the wireless sensor technology also emerged as powerful technology. Congestion is the major issue on which many research has been done. Typically in Event driven WSN the data that is generated is of great importance. The paper presents an efficient way to collect the data, which is sent through the Event Driven network. The results show that the mobile data collector node efficiently collects the data from the event generated area and disseminates it to sink node. For this a Mobile data collector algorithm is proposed. The mobile data collector node will follow some fix predefined path or random path to disseminate packet to destination node. The experimental results show that the reliability of network is increased.
\end{abstract}

\section{Keywords}

Wireless Sensor Network, Congestion, mobile data collector node, Event driven system.

\section{INTRODUCTION}

When many sensors cooperatively monitor large physical environment, they form a Wireless Sensor Network (WSN). Sensor nodes not only communicate with each other but also communicate with their base station (BS) using their wireless radios, allowing them to disseminate their sensor data to the remote processing ,visualization, analysis, and Storage system. The sensor communicates with each other using broadcast mechanism. Whenever an event is generated the Sensor nodes sense the event and broadcast the event information to the Sink node to take appropriate action. the wireless sensor network can be classified as Event Driven or periodic data generation.

EWSN (Event Driven Wireless Sensor Network) network is a Wireless Sensor Network that is driven by the generation of the event information. The event information that is generated must be transmitted immediately without any loss of information. This information helps the Sink node to take the necessary action on the Event. For e.g. if there is occurrence of event Fire, the nodes will detect the event and send it to Sink node to take necessary action.

Reliable data transmission is the important requirement of wireless network. To maintain the reliability the network, the data transmitted in the network from source to destination must reach without loss. Congestion plays an important role in maintaining reliability. If the nodes are congested then there is loss of data packets.

In EWSN based network, when the event is generated, it is detected by the nodes surrounding it and all of these nodes will make a decision about occurrence of event. This event information is transmitted to sink node to take appropriate action. Here there is chance of congestion at the nodes that are one hop away from the sink nodes. Many solutions have been proposed to avoid the congestion at the nodes that are one hop away from source node. There are two mechanisms to control the congestion. In first method, it will tell the sender to slow down the sending data packet rate (source rate adjustment) and another is retransmitting the lost data packet, so that reliability can be maintained. For this the some advancement is proposed by adding the mobile data collector node that will carry the data packets towards the sink node and reduces the energy consumption of node and making reliable transmission of data in the network.

The work is organized as follows. In section 2 the Literature Survey for the topic is conducted which describes the work associated with the topic. In section 3 the proposed system is described, defining the basics requirement and then proposing the algorithm for the same, which is followed by Conclusion and References

\section{LITERATURE SURVEY}

ESRT (Event-Sink Reliable Transport), is a protocol that is developed by O.B. Akan.it is designed to achieve reliable event detection by adjusting the rate at which the sender sends the data. ESRT also adjusts the reporting frequency of node such that the observed event reliability is higher than actual desired value which avoids congestion. The observed event reliability is defined as the number of packets received at the sink. The drawback of this method is the self-adjusting method where all sensor nodes are controlled at once, which treats all the regions of interest in the same way as uninteresting regions. Also, the feedback and rate control scheme in ESRT causes in considerable extra delay.

The paper [8] surveys the various root causes of congestion in WSN.it analyze the root cause of congestion as Input and Output Flow rate, Node Density, Unbalanced Distribution of Load, Service Time of Node and Reliability of Network. The survey helps to achieve more reliable network. SPEED [7] protocol is designed to provide end-to-end deadline guarantees for real-time packets in sensor networks, which is very suitable for emergent information dissemination in EWSNs. In SPEED protocol, each node maintains neighbor node information such as geographic distance and average delay to each neighbor. Using the distance and delay, each node could evaluate the packet progress speed of each neighbor node and forwards a packet to a node whose progress speed is higher than the pre-specified lower-bound speed. By this way, the sensor node prefers to choose the faster node as forwarder and shorten the delay further. However, the SPEED protocol provides only one networkwide speed, which is not suitable for differentiating various traffics with different deadlines. 
In the Wireless Sensor Network, depending upon the application scenario, the phenomenon could be classified into global phenomenon (GP) and local phenomenon (LP). In order to reduce the transmissions to minimize the power consumption, each node makes a local decision considering its own observation and also the decision made by the previous node. To minimize the error probability, a simple fusion rules for both the global and local phenomenon scenario are proposed. However, the scheme in [3] is only suitable for a chain network, which is not scalable for more complex network topology. In EWSNs, whether the event occurs or not is more of interest to users rather than the detailed event information.

In the work of [4], three detection schemes have been investigated: the centralized, distributed and quantized scheme. A centralized scheme requires each sensor node to forward all its observations to the fusion center, which results in large energy in communication. A distributed scheme, on the other hand, allows each sensor node to make its own decision and then send out only its 1-bit decision. This reduces communication energy at the cost of increased processing energy and reduced detection accuracy. In a quantized scheme, each node processes observations data and sends a quantized M-bit quantity to the fusion center, and the control center makes the final decision based on the $\mathrm{K}$ quantized quantities. Composite event is first proposed in [5] to make accurate event detection. To detect the occurrence of the event, the event must be detected by at least $\mathrm{K}$ sensors, where $\mathrm{k}$ is a user-defined parameter. A loss tolerant reliable data transport mechanism for dynamic Event Sensing (LTRES) was proposed in [7], which focuses on the reliable event information transmission by appropriate congestion control scheme. A distributed source rate adaptation mechanism is designed, incorporating a lightweight congestion control mechanism with loss rate as the indication of upstream congestion level. By this way, the traffic injected into the network is regulated, so that the reliability requirement can be satisfied. However, the timeliness requirement is not considered in LTRES. The [1], works out to avoid the congestion by making a single decision.it proposes the EEDP (efficient event detection protocol) to detect efficiently the generation of the event. for this it makes the use of concept of atomic event and composite event. When an event (eg. Fire) is sensed by a number of sensors such as smoke sensor, fire sensor the atomic event of it generated. The node locally broadcast to its neighbor node its reading. By making use of these multiple atomic values, the node forms the composite event which is a single bit of information. This composite event is broadcasted to the Sink node through the intermediate neighboring node which uses the multi copy scheme to avoid the loss of the packet near the sink node. Many research works have shown that there is chance of congestion at the nodes that are one hop away from the Sink node. So to avoid the loss of packets near the Sink node the paper proposes a solution of multicopy Scheme. The Drawback of the paper can be thought as it continuously broadcast the packet for the specified time period and by using multicopy scheme it maintains the single alarm packet into its buffer until the packet reaches the destination and an acknowledgement is received. Energy of sensor node is wasted, during this process. So a new modification is proposed in the paper.

The Simulation Tool NS-2 is used to evaluate the proposed algorithm. The simulation parameters chosen are shown in the Table1.

\section{Table I : Simulation Parameters}

\begin{tabular}{|l|l|}
\hline Parameters & Values \\
\hline Simulation Time & $200 \mathrm{~s}$ \\
\hline Monitoring Area & $200 * 200$ \\
\hline Number of Nodes & 100 \\
\hline Communication Range & $40 \mathrm{~m}$ \\
\hline MAC Layer & IEEE 802.11 \\
\hline Length of Packet & 1000 bytes \\
\hline Location of Sink & Center \\
\hline
\end{tabular}

The network considered has a total of 100 nodes with a single sink. The nodes are uniformly distributed randomly within $200 \mathrm{~m} * 200 \mathrm{~m}$. The communication range is 40 meters and sink is located at center. The hardware configuration requires processor of $1.7 \mathrm{GHz}$, and Software Ubuntu, ns-2.29 or later version, TCL file and $\mathrm{C}++$.

\section{SYSTEM MODEL}

A EWSN network consists of a number of different sensor nodes equipped with different sensors and a sink node as shown in Fig. 1. Nodes which appear in the radius of an event sense the event and generate value independently and collect Observation data from its neighbor node. Now all the nodes present in range of event area will make a local event decision and this decision is then combined to make a composite decision which is given to the MDC node which carries it towards the Sink node to take further action.

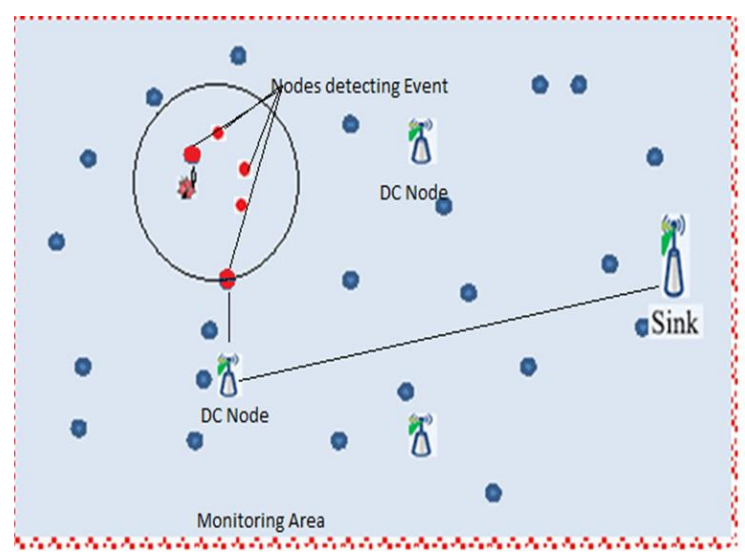

\section{Fig 1: EWSN system with Mobile data collector node}

As mentioned in reference [1], we make use of concept of Atomic event and composite event to mark the generation of event.

Atomic Event: The necessary condition of atomic event is

$$
\begin{aligned}
& \mathrm{x}_{\mathrm{i}}^{\mathrm{m}}>\Gamma_{\mathrm{i}}^{\mathrm{m}} \text { then } \\
& \mu_{\mathrm{i}}{ }^{\mathrm{m}}=1 \text { else } \mu_{\mathrm{i}}{ }^{\mathrm{m}}=0
\end{aligned}
$$

where 
- $\mathrm{x}_{\mathrm{i}}^{\mathrm{m}}$ is observed value of sensor node

- $\mu_{\mathrm{i}}^{\mathrm{m}}$ is Atomic value is set either 1 or 0 depending upon occurrence of event.

- $\quad \Gamma_{\mathrm{i}}^{\mathrm{m}}$ is the threshold value which is set to some value depending upon the sensor type and beyond which if the value goes then there is some abnormal event happened.

Table II: Symbols used

\begin{tabular}{|c|c|}
\hline Symbol & Use \\
\hline $\mathrm{N}$ & Number of Nodes \\
\hline $\bar{D}(\mathrm{i}, \mathrm{j})$ & Distance of node $\mathrm{i}$ from $\mathrm{j}$ \\
\hline $\mathrm{M}$ & Number of components of sensor node \\
\hline $\mathrm{N}_{\mathrm{i}}$ & Set of neighbor node of $i$ \\
\hline$x_{i}^{m}$ & the analog observation of mth sensor node $\mathrm{i}$ \\
\hline$\mu_{\mathrm{i}}^{\mathrm{m}}$ & local atomic decision of Sensor \\
\hline$\Gamma \mathrm{im}$ & $\begin{array}{l}\text { the threshold value of node } i \text { for the mth sensor } \\
\text { of node } i\end{array}$ \\
\hline$\Delta$ & a composite event \\
\hline B & $\begin{array}{l}\text { the threshold range of the mobile data collector } \\
\text { node }\end{array}$ \\
\hline
\end{tabular}

Composite Event: The Composite Event is formed as

$\Delta=\mu_{\mathrm{i}}^{\mathrm{m}} 1$ AND $\mu_{\mathrm{i}}^{\mathrm{m}} 2$ AND $\ldots \ldots \mu_{\mathrm{i}}^{\mathrm{m}} \mathrm{n}$

If $\Delta=1$ then Event Occurred, the node will broadcast the occurrence of event for time $t$

Else $\Delta=0$ then Event has not occurred, it will reset the values of $x_{i}^{m}$

The proposed System makes use of the concept of the Composite Event which is the anding of all the sensed values of the sensor node.

\subsection{Mathematical Model}

Consider a static event-driven wireless sensor network with $\mathrm{N}$ sensor nodes. It is assumed that each node can communicate with each other. Some notations and symbols used in paper are shown in table2. Here as each node can communicate with each other there is a symmetric relation between them. Consider a matrix, which will contains entry as 1 if node $i$ can transmit to node $\mathrm{j}$ which also means that $\mathrm{j}$ can communicate with i.

\section{Neighbor Set (NS):}

This set is used to indicate the nodes which are in interference range of the node, to make a composite decision.
2. MDC set (MS):

The Mobile Data Collector (MDC) set is used by the mobile data collector node, which it uses to trace path to reach the node who has broadcasted the data. It calculates the distance by Euclidean formula and maintains the list of it into it.

$\mathrm{D}(\mathrm{i}, \mathrm{s})<\mathrm{D}(\mathrm{j}, \mathrm{d})$

$\mathrm{D}(\mathrm{i}, \mathrm{s})>\mathrm{D}(\mathrm{j}, \mathrm{d})$

Dist $\mathrm{D}=\sqrt{(\mathrm{x} 2-\mathrm{x} 1)^{2}+(\mathrm{y} 2-\mathrm{y} 1)^{2}}$

When a node detects the atomic event it will broadcast its information locally to the neighboring node. The neighboring node similarly broadcast the information to each other. Now when each node receives its value then it will perform the logical AND operation to form Composite Event. If the output of this operation is 1 then the event has been detected else if it is 0 then there is no occurrence of event. When the event has been detected then the node will broadcast the corresponding event information

The algorithm proposed for the same are local broadcast algorithm and Data collector algorithm which are given as :

O Input: The observation of node $\mathrm{i}: \mathrm{x}_{\mathrm{i}}^{\mathrm{m}}$,

O Output: Event information is sent to sink

Step 1: Node senses the generation of event.

Step 2: when the sensed value goes above the threshold value i.e. $\mu_{\mathrm{i}}^{\mathrm{m}}=1, \mu_{\mathrm{i}}^{\mathrm{m}}$ is set to 1 and the node detects the occurrence of event.

Step3: the node then forwards the packet to the mobile data collector node. The MDC node will follow the following steps

Step 4: The Mobile $\stackrel{\bullet}{ }$ Dhile $\mathrm{BS}<=\beta$ do broadcasted message

Step 5: When it receives the MSG it will find the address of the source sending the message from the packet

Step 6: From the address database it will find out the path to reach the Source node

Step 7: The address database is calculated for each pair of sensor node by using the Euclidean Distance

$\mathrm{D}=\sqrt{(\mathrm{x} 2-\mathrm{x} 1)^{2}+(\mathrm{y} 2-\mathrm{y} 1)^{2}}$

Step 8: After finding the Path, it makes use of random way to reach the source node

Step 9: after collecting data, again it makes use of random way to reach the destination

Step10: if Buffer Size (BS) of MDC node is not full, it will collect other Event information on the way if it is present.

End while.

Step 11: when $\mathrm{BS}>\beta$ then it will broadcast it contents to replica MDC node 
The data collector Node makes uses of the Reactive Routing Protocol AODV. An On-Demand Distance Vector Protocol is used, which will keep the neighbor distance location $\mathrm{x}$ and $\mathrm{y}$ coordinates and Delay.

The Results of the experiment simulation performed are given as follows. The comparison of packet delivery ratio, total packets sent, total packets received, total packets dropped, along with end to end delay is performed. The simulation is carried out for 100 nodes. A random topology is created with AODV protocol. The transmission range is $40 \mathrm{~m}$.

The graph for total packets sent and received in simulation using EWSN system without MDC node and EWSN based mobile data collector node is given as

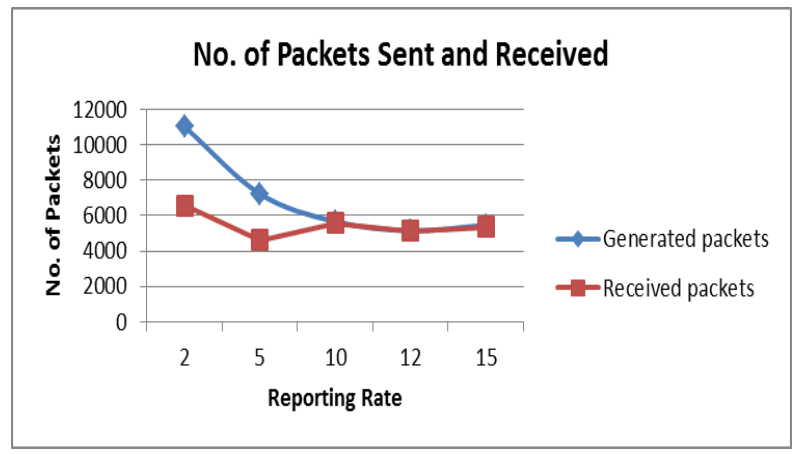

Fig 2:No. of packets sent and received without MDC node

The graph for total packets sent and total packets received in EWSN system using MDC node is given as

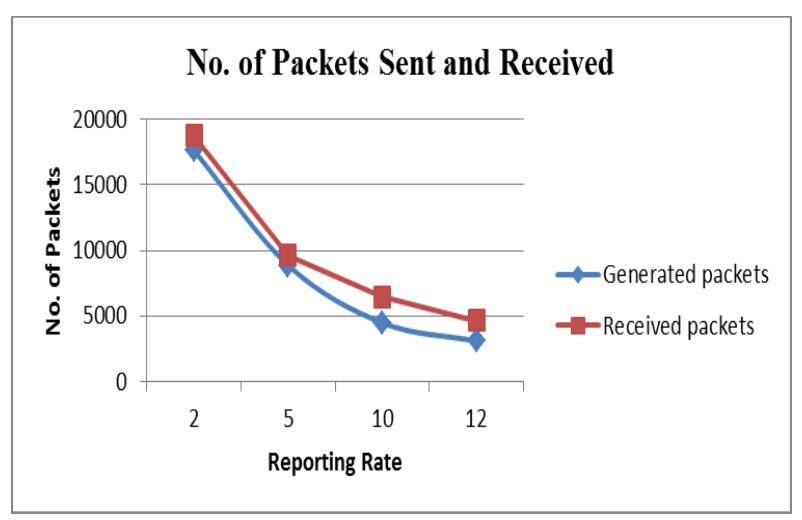

Fig 3: No. of packets sent and received with MDC node

From the graph it is clear that the total packets that were received in EWSN system with mobile data collector node are high as compared to EWSN system with GPSR code.

Similarly the comparison graph for the packet delivery ratio can be given as

\section{PDR with reporting rate 10 and Packet size} 512

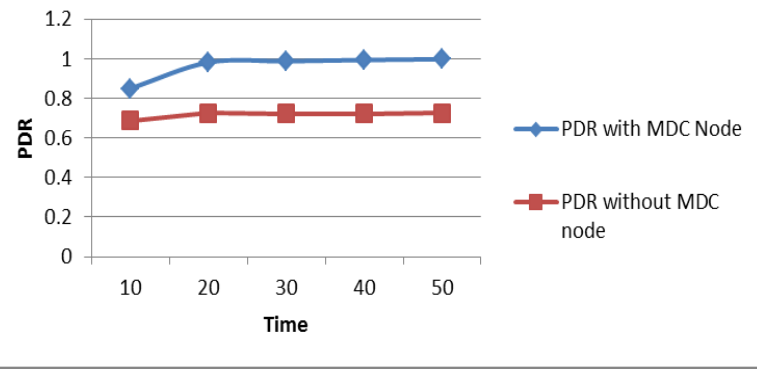

Fig 4: Comparison of PDR for MDC node and without MDC node

From the graph it is clear that, the EWSN system gives the relatively good packet delivery ratio

Comparison of End to end delay for EWSN system with GPSR code and EWSN system with mobile data collector node is given as

\section{e2e delay with reporting rate 10 and packet size 512}
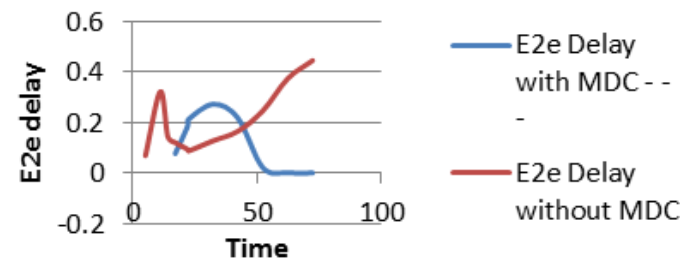

Fig 5: Comparison of e2e delay

From the results above, it is found that the Mobile data collector node can achieve high reliability and less loss of data packet as compared to existing EWSN system with GPSR.

The graph shows the energy consumed by all nodes for the EWSN architecture with MDC node and without MDC node. In this graph for all nodes initial energy is set as 40 joules. after simulation the average energy of all nodes is calculated. It is found that energy consumed by EWSN system without MDC node model is more than with EWSN system model. The energy consumed is less in our model as no node is involved in transmitting packets directly towards the sink node or through the intermediate node. 


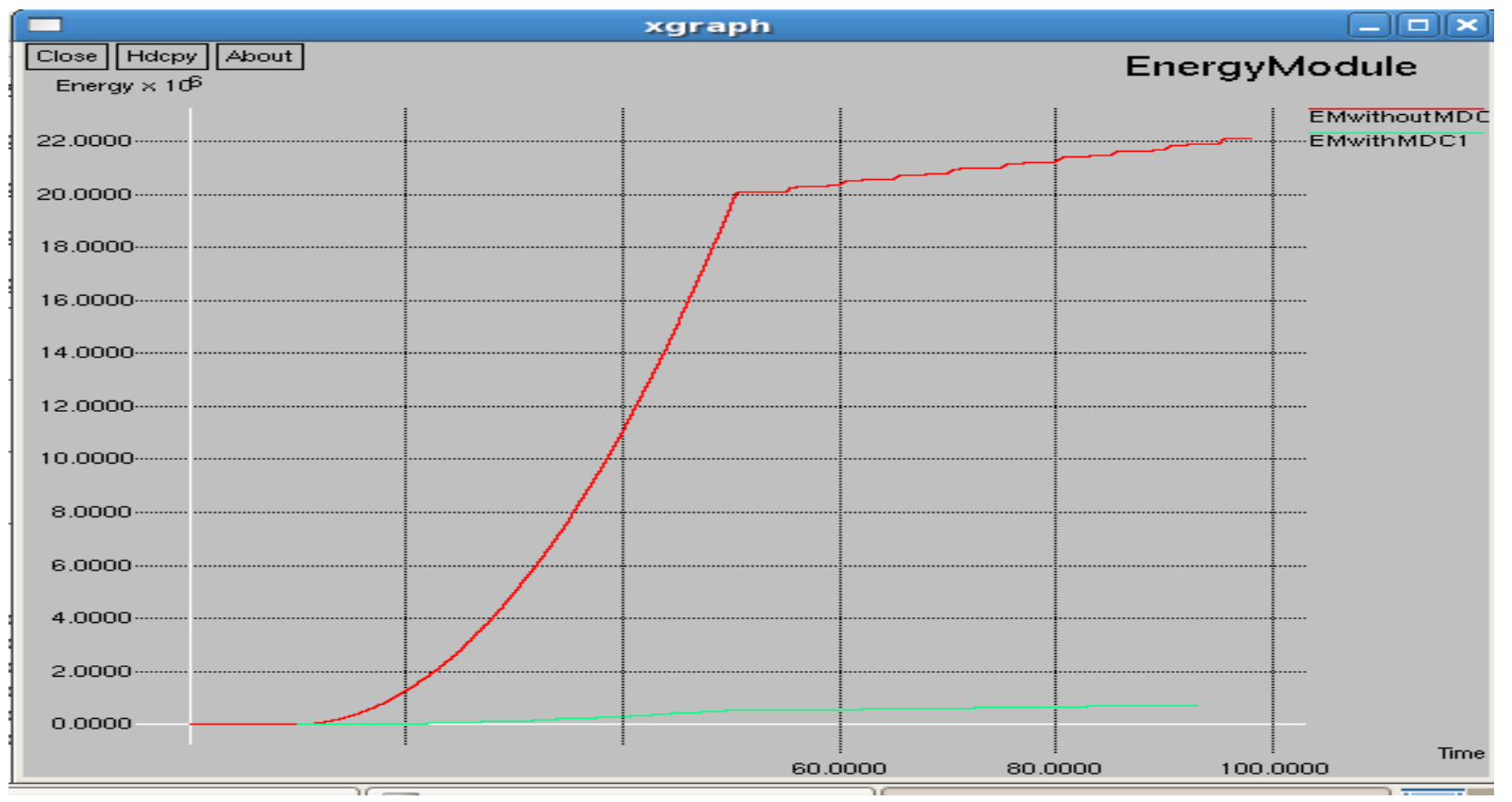

Fig 6: Energy Graph

\section{CONCLUSION}

Most of the wireless sensor network tries to maintain the challenging issue of Reliability by either source rate adjustment or dealing with data packets after the congestion scenario occurs. i.e. loss recovery. Here using Energy Efficient and Reliable Data collection in EWSN based Wireless Sensor Network, it is tried to maximize the data packets that are received at the sink node by presenting the concept of Mobile data collector node. Thus the throughput as well as packet delivery ratio increases and can finally improve the reliability of network.

The concept of Mobile Data Collector Node in Wireless Sensor Network can help to increase the network life time by avoiding the loss of packets present at the nodes that are one or two hop away from the sink node. As too many packets arrive near the sink node the energy of the node falls considerably, this leads to loss of the packets. Thus if the Mobile data collector node is used, then it will carry the data packets towards the sink node, without consuming too much energy of nodes that are near to sink node. Hence it can be proved that, the mobile data collector node improves the reliability of the network and increase the network lifetime by reducing the energy consumption.it also helps to maintain the sleep wake state of nodes.

\section{REFERENCES}

[1] Lulu Liang, Deyun Gao, Hongke Zhang, and Oliver W. W. Yang, "Efficient Event Detecting Protocol in EventDriven Wireless Sensor Networks", IEEE SENSORS JOURNAL, VOL. 12, NO. 6, JUNE 2012.
[2] O. B. Akan and I. F. Akyildiz, "Event-to-sink reliable transport in wireless sensor networks", IEEE/ACM Trans, Netw., vol. 13, no. 5, pp. 1003-1016, Oct. 2005.

[3] Lucchi and M. Chiani, "Distributed detection of local phenomena with wireless sensor networks", in Proc. ICC, Cape Town, South Africa

[4] L. Yu and A. Ephremides, "Detection performance and energy efficiency trade-off in a sensor network,", in Proc. Allerton Conf., Allerton, IL, Oct. 2003, pp. 390-399

[5] A. V. U. P. Kumar, A. M. V. Reddy, and D. Janakiram, Distributed collaboration for event detection in wireless sensor networks, in Proc. MPAC, Grenoble, France, Nov. 2005, pp. 1-8

[6] T. He, J. Stankovic, C. Lu, and T. Abdelzaher, Speed: A stateless protocol for real-time communication in sensor networks, in Proc. 23rd Int. Conf. Distr. Computer. Syst., May 2003, pp. 46-55

[7] C Y. Xue, B. Ramamurthy, and Y. Wang, Providing reliable data transport for dynamic event sensing in wireless sensor networks, in Proc. ICC, Beijing, China, May 2008, pp. 3 146-150.

[8] Vivek Deshpande, Prachi sarode, Root Cause Analysis of Congestion in Wireless sensor networks, in Proc. 23rd Int. Journal of Computer Applications. Syst., 2010, vol1No.18 